\title{
Accuracy of shear wave elastography in characterization of thyroid nodules in children and adolescents
}

Mohammed Hazem 1,2, Ossama M. Zakaria 1,3, Mohamed Yasser Ibrahim Daoud', Ibrahim Khalid Al Jabr 1*, Abdulwahab A. AlYahya ${ }^{1,4}$, Ahmed Gaber Hassanein ${ }^{5,6}$, Abdulrahim A. Alabdulsalam7, Mohammed Qasem AlAlwan ${ }^{1,8}$ and Nahla Mohamed Ali Hasan ${ }^{9,2}$

\begin{abstract}
Background: Thyroid nodules are an important health problem in children and adolescents. They possess a higher risk of malignancy in comparison to adults. This fact forms a great dilemma for clinicians. The aim of this study was to evaluate the reliability of shear wave elastography (SWE) as a non-invasive technique in the characterization of thyroid nodules in children and adolescents.

Methods: This prospective study included 56 patients with thyroid nodules. All the patients underwent clinical assessment, laboratory investigations, ultrasound, and Doppler examination, followed by an SWE assessment. Statistical analysis was performed and the best cut-off value to differentiate benign from malignant nodules was determined using the ROC curve and AUC.

Results: Seventy-two nodules were detected in the examined patients (ages ranged from 11 to 19 years, with mean age of $14.89 \pm 2.3$ years). Fifty-eight nodules (80.6\%) were benign, and fourteen nodules (19.4\%) were malignant (histopathologically proved). Highly suspicious criteria for prediction of malignancy by ultrasound and Doppler were hypoechoic echopattern, internal or internal and peripheral vascularity, microcalcifications, taller-than-wide dimensions, irregular outlines, and absence of halo $(p<0.05)$. The diagnostic performance for their summation was $70.69 \%$ sensitivity, $82.8 \%$ specificity, $80.45 \%$ accuracy, a $63.79 \%$ positive predictive value (PPV), and $87.9 \%$ negative predictive values (NPV). Regarding SWE, our results showed that $42.2 \mathrm{kPa}$ was the best cut-off value, with AUC $=0.921$ to differentiate malignant from benign nodules; the diagnostic performance was $85.71 \%$ sensitivity, $94.83 \%$ specificity, $93.06 \%$ accuracy, 76.9\% PPV, and 93.2\% NPV.
\end{abstract}

Conclusion: Shear wave elastography is a non-invasive technique that can assist in the diagnosis of malignant thyroid nodules among children and adolescents.

Keywords: Ultrasound, Shear wave elastography, Thyroid nodules, Children and adolescents

\footnotetext{
*Correspondence: ialjabr@kfu.edu.sa

${ }^{1}$ Department of Surgery, College of Medicine, King Faisal University, P.O.

Box: 400, Al-Ahsa 31982, Kingdom of Saudi Arabia

Full list of author information is available at the end of the article
}

\section{Key points}

- Shear wave elastography (SWE) is an innovative technique providing new insights into thyroid nodules characterization.

- Malignant thyroid nodules have higher SWE indices (stiffer) than benign nodules. 
- SWE has better diagnostic performance than ultrasound and Doppler imaging.

\section{Introduction}

Thyroid nodules in children pose a significant problem and dilemma for endocrinologists, surgeons, and radiologists in regard to their pathological nature $[1,2]$. In general, thyroid nodules in adults are more likely to be benign rather than malignant. However, among children and adolescents, the chances of a nodule being malignant is higher in comparison to adults [3]. Many patients with thyroid malignancy present with local metastasis in the cervical lymph nodes. This might be attributed to delay in diagnosis. However, distant metastasis is not as common [4].

Many diagnostic tools exist for evaluating thyroid nodule/s. The universally accepted protocol includes a thorough clinical examination, laboratory investigations, thyroid sonography, as well as fine-needle aspiration biopsy (FNAB) [2].

There is a debate on which is the best tool to investigate thyroid nodules. Sonography is still considered to have a higher range of diagnostic sensitivity and specificity compared to clinical examination [5]. However, ultrasound features of thyroid nodules in children are not strong predictors of malignant or benign etiology of thyroid nodules [6]. FNAB is currently considered one of the most accurate techniques to assess the pathological nature of thyroid nodules. However, it may be difficult to perform in children [7].

The last decade has witnessed the increasing use of sonoelastography (SE) as an additional tool in the assessment of thyroid nodules [8]. SE relies on the concept that malignant tumors are usually stiffer and firmer compared to their benign counterparts [9]. Earlier SE techniques depended on assessing tissue stiffness by applying strain/ pressure upon the examined tissue; however, this technique does entail some limitations. These include low reproducibility, lack of quantitative assessment of tissue stiffness, as well as being operator dependent [10].

Shear wave elastography (SWE) is a relatively newly developed SE technique that can quantitatively evaluate the examined tissue for its stiffness. It measures elasticity by tracing the shear wave propagation through tissues to provide quantitative measurements. It has been reported to be more reproducible and less operator dependent in comparison to SE $[11,12]$. The use of SWE for quantitative assessment of the elasticity of adult thyroid nodules has been previously reported. These reports showed that quantitative elastographic evaluation of thyroid nodules was significantly different between malignant and benign nodules [13-15]. Normal thyroid tissue elasticity in children was previously assessed [16]. However, very few studies have reported the use of elastography to assess thyroid nodules in children $[17,18]$, and, to the best of our knowledge, none have yet studied the use of SWE specifically for differentiating benign and malignant thyroid nodules in the pediatric population.

The current study aims to evaluate the reliability of SWE in characterizing thyroid nodules among children and adolescents through correlation with FNAB and histopathological results. Additionally, the study aims to establish a cut-off value to distinguish between benign and malignant thyroid nodules.

\section{Methodology \\ Study design}

This prospective study took place over a period of five years from June 2015 to May 2020. Ethical approval was obtained from our intuitional review board (IRB), and informed written consent was obtained from patients' parents or legal guardians. All techniques were performed in accordance with the Helsinki Declaration 1975 , as revised in 2013. The study included 56 children and adolescents referred from relevant outpatient clinics who clinically presented with nodular thyroid enlargement. Exclusion criteria included patients with completely cystic nodules, nodules with coarse calcifications, and patients that refused to do FNAB or surgical excision in cases with indeterminate FNAB results (which is considered the golden standard for diagnosis).

\section{Assessment of patients}

All the patients were interviewed for clinical assessment and laboratory investigations, followed by multiple radiological examinations that included chest X-ray, abdominal ultrasound, and different thyroid ultrasound techniques, including B-mode ultrasound, Harmonic ultrasound, Doppler ultrasound, and SWE examination. FNAB was performed for all cases, and the final diagnosis was based on cytopathologic results in addition to histopathologic results of the surgically resected specimen. Moreover, all proved to be benign nodules by cytopathological, or histopathological analysis; they were subjected to clinical and ultrasound follow-ups to exclude any possibilities of false negative results.

\section{Conventional and Doppler ultrasound examination}

Ultrasonographic examination was performed by a single expert radiologist who has an experience of 16 years in thyroid ultrasound and Doppler examination, using a Philips EPIQ 7G ultrasound machine (Philips, USA) with a 7-12 MHz broadband linear array transducer. Patients were examined in supine position with elevated shoulders by small pads to facilitate full extension of the neck 
that leads to complete exposure of the lower margins of the thyroid glands. Scans of the gland were obtained in both longitudinal and transverse planes. All thyroid lesions were initially assessed by B- mode and Doppler ultrasound.

The following parameters were assessed in each nodule, as recommended by the American Thyroid Association (ATA) guidelines: size; shape; margin; echogenicity (hypoechoic, isoechoic, and hyperechoic); internal content (completely solid or partially solid); presence or absence of calcifications, and, if present, its size was determined as microcalcification when $<1 \mathrm{~mm}$ or macrocalcification $>1 \mathrm{~mm}$; and the presence of absence of a halo sign around the nodule (Fig. 1). Furthermore, the nodules were classified into two types according to their vascular patterns as assessed by Doppler ultrasound: Type 1: Absent blood flow within the nodule or the blood flow present only at the periphery of the nodule; Type 2: Blood flow detected within the center of nodule or within the center and around the nodule (Fig. 2).

Moreover, we implemented sonographic assessment of the internal cervical chain of the lymph nodes. This technique was significantly valuable in differentiating the hyperechoic and punctate calcifications due to papillary carcinoma from other malignant cervical lymphadenopathies [19].

\section{SWE examination}

After completing ultrasonographic examination, SWE examination was performed during the same sitting by the same radiologist, who has five years of experience in SWE examination. The transducer was placed in a sagittal plane to avoid motion artifacts generated by the trachea and carotid arteries. Patients were asked to hold their breath and to not swallow for a short time while the image of the SWE was formed. The transducer was held in a stable position without performing any compression over the gland to minimize the artifacts resulting from compression (free hand technique), then SWE was started upon the conventional sonographic image.

The color box with adequate size was applied and displayed as an area of multiple colors, with blue representing softer tissue and red representing harder tissue. Images were obtained when there were no artifacts.

Quantitative elastographic assessment was performed using a $2 \times 2 \mathrm{~mm}$ region of interest (ROI), with the transducer positioned at the stiffest area, avoiding cystic areas, calcification, and normal thyroid tissue (Figs. 3, 4). All measurements were recoded and saved in kilopascal $(\mathrm{kPa})$. Three measurements were taken for each nodule: the minimum, maximum, and mean SWE. The examination was repeated three times for each lesion, and the
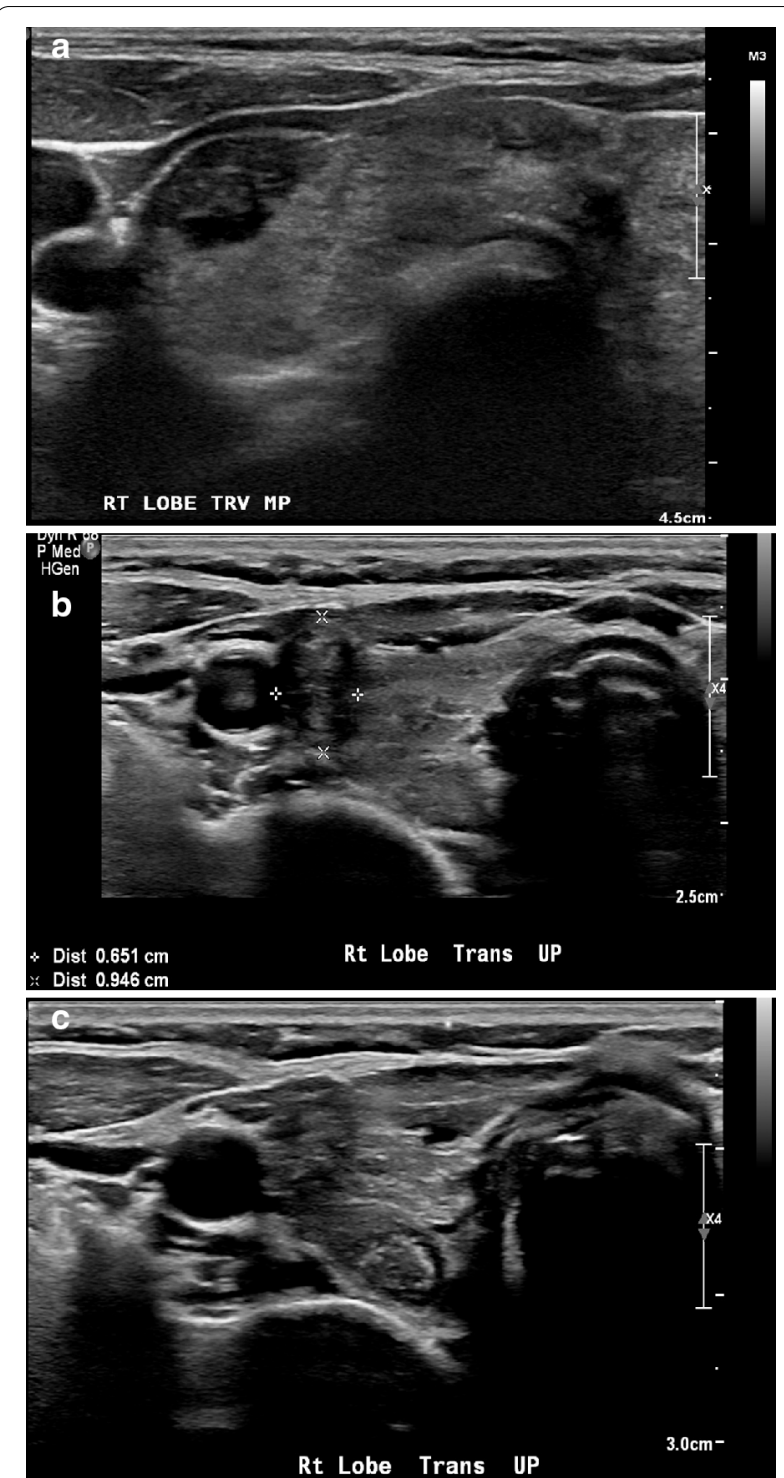

Fig. 1 Multiple transverse B-mode ultrasound images obtained by linear probe examination showing: a Well-defined hypoechoic nodule with irregular outline within the right lobe of the thyroid gland. b Well-defined non-homogenous hypoechoic, taller-than-wide; smooth outline nodule is seen within the right thyroid lobe. It is not surrounded by a halo and does not contain internal calcification. c Well-defined isoechoic nodule surrounded by hypoechoic halo with regular outline within the posterior aspect of the right lobe of the thyroid gland

average of the three measurements was used as the final result.

Fine-needle aspiration biopsy (FNAB) and histopathologic examination

Ultrasound-guided FNAB was taken from all patients for cytopathologic examination. The slides were stained 

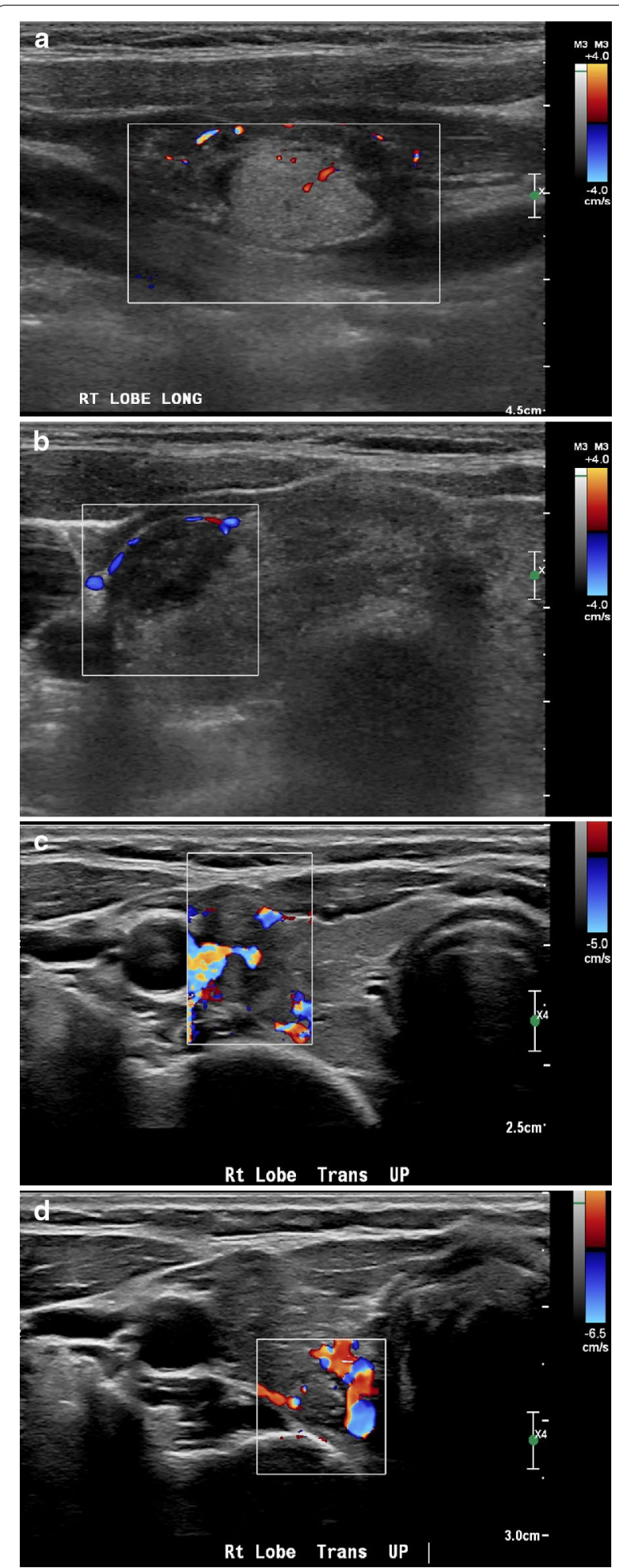

Fig. 2 Multiple transverse and longitudinal Doppler ultrasound images performed by linear probe showing: a A hyperechoic nodule with internal vascularity seen within. $\mathbf{b}$ A hypoechoic nodule with peripheral vascularity seen surrounding it. $\mathrm{A}$ non-homogenous isoechoic nodule with mixed peripheral and central vascularity. $\mathbf{d} A$ hyperechoic nodule with mixed peripheral and internal vascularity

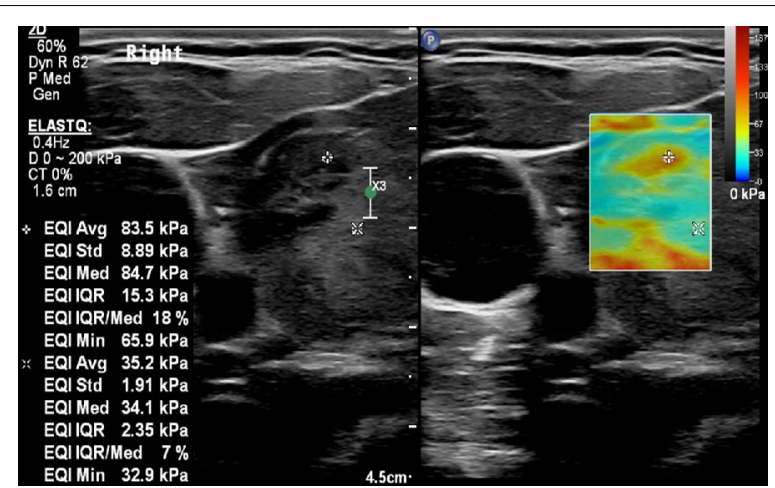

Fig. 3 A 14.5-year-old female patient with FNAB-proven papillary thyroid carcinoma. Transverse scan for left lobe of the thyroid gland obtained by linear probe. On the right side of the figure, grayscale US shows a hypoechoic nodule with irregular margin which was assessed as a suspicious nodule on grayscale US. On the left side of the figure, SWE displays a heterogeneous color elasticity signal with central high SWE areas (red); mean SWE was $83.5 \mathrm{kPa}$

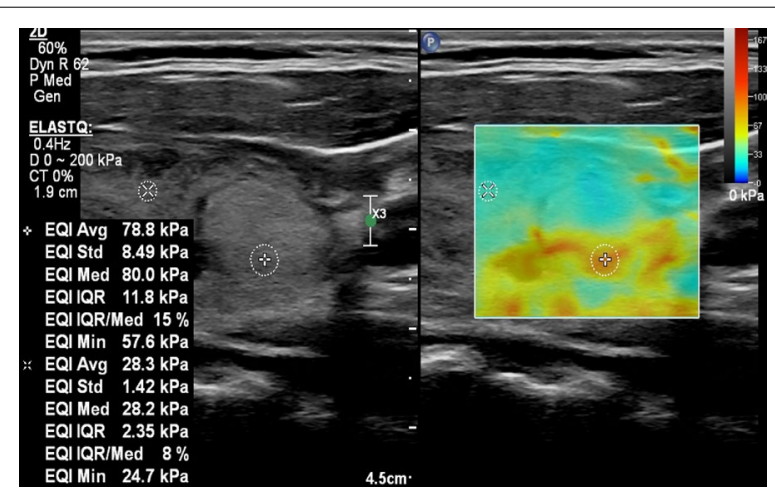

Fig. 4 A 16-year-old male patient with FNAB-proven focal nodular hyperplasia. Transverse scan for right lobe of the thyroid gland performed by linear probe. On the right side of the figure, grayscale US shows a hyperechoic nodule with a well-defined margin. On the left side of the figure, SWE displays a heterogeneous color elasticity signal with peripheral high SWE areas (red)

with pap stain and a Romanowsky-type stain, and it was interpreted by a single expert pathologist. Reporting of FNAB results followed the Bethesda system for reporting thyroid cytopathology [20]. For those cases with indeterminate results (i.e., Category III and IV according to the Bethesda system) and who had surgical excision of the nodule performed, the final diagnosis was determined through histopathologic examination of the excised nodule.

\section{Statistical analysis}

Data coding, entry, and analysis was accomplished with the aid of the SPSS ${ }^{\circledR}$ program (version 22) together with MedCalc ${ }^{\circledR}$ software. Descriptive statistics in the form of 
numbers and percentages were used for qualitative variables, while mean and standard deviation were used for quantitative variables.

For the detection of association and/or links between categorical grayscale ultrasonic characters and nature of thyroid nodules whether benign /malignant, a Chisquare test was used, and, when indicated, the Fisher exact test was used (with expected cell count less than 5). The odds ratio was calculated to assess these associations and the Phi co-efficient was calculated to determine the effect size. For these ultrasonic characters, the following were also calculated: the sensitivity, specificity, accuracy, PPV, and NPV.

The independent samples t-test was used to detect differences between malignant and benign nodules regarding quantitative SWE measures. For these quantitative SWE measures, a receiver operating characteristic curve was used to assess AUC, cut-off values, sensitivity, and specificity, in addition to calculating accuracy, PPV, and NPV. The accepted level of significance for all analyses was stated at 0.05 (two-tailed $p$ value of $<0.05$ was considered significant).

\section{Results}

\section{Patients}

Fifty-six patients were included in the study. They included 36 females and 20 males with a ratio of 1.8 to 1. Patients' ages ranged from 11 to 19 years, with a mean age of $14.89 \pm 2.3$ years. Out of the 56 patients; 42 (75\%) presented with a single nodule, while the remaining 14 (25\%) had more than one nodule. The total number of nodules from all patients was 72 .

\section{Pathological findings}

Cytopathologic assessment along with histopathologic evaluation of excised nodules was considered as the main diagnostic method for the differentiation between benign and malignant nodules. On FNAB assessment, out of the 72 nodules, $51(70.8 \%)$ were benign, $9(12.5 \%)$ were malignant, and 12 (16.7\%) showed indeterminate results. Cases with indeterminate FNAB results underwent surgical excision of the nodule, and the histopathologic assessment in these cases showed a benign result in seven cases and a malignant result in five cases. Therefore, fifty-eight (80.6\%) nodules were benign, whereas 14 nodules (19.4\%) proved to be malignant. All the malignant nodules were diagnosed as papillary carcinoma $(n=14)$. The majority of benign nodules were diagnosed as nodular hyperplasia/benign follicular nodules $(n=51,87.9 \%)$. The remaining seven nodules $(12.1 \%)$ were benign nodules associated with lymphocytic thyroiditis/Hashimoto thyroiditis (Table 1).
Table 1 FNAB and postoperative histopathology findings of thyroid nodules ( $n=72$ nodules in 56 patients)

\begin{tabular}{lll}
\hline $\begin{array}{l}\text { FNAB and histopathology } \\
\text { findings }\end{array}$ & Number & \\
\hline Benign & $\begin{array}{l}\text { Nodular hyperplasia/Benign fol- } \\
\text { licular nodule }\end{array}$ & 51 \\
& $\begin{array}{l}\text { Benign nodule associated with } \\
\text { lymphocytic thyroiditis }\end{array}$ & 7 \\
& Total & 58 \\
Malignant & Papillary carcinoma & 14 \\
Total & & 72 \\
\hline
\end{tabular}

\section{Conventional ultrasound and Doppler results}

The mean size of thyroid nodules (according to the longest diameter) was $(1.8 \pm 0.68 \mathrm{~cm}$; range: $0.8-$ $3.2 \mathrm{~cm}$ ) with no statistical significance between benign and malignant nodules. The other sonographic findings of the 72 nodules are variable and are mentioned in Table 2.

The sonographic criteria which were commonly observed with malignant nodules included hypoechoic echopattern (observed in 71.4\%), internal or internal and peripheral vascularity (85.7\%), microcalcification (78.6\%), and not surrounded by halo (85.7\%). These criteria were statistically significant compared to their benign counterparts $(p<0.001$ in all). Moreover, the malignant nodules were taller rather than being wide (64.3\%) and had an irregular outline (57.1\%); all these findings were statistically significant in comparison with benign nodules $(p<0.05)$ (Table 2$)$.

The obtained statistically significant sonographic criteria were compared with pathological findings for the assessment of their diagnostic performance (sensitivity, specificity, accuracy, positive predictive value, and negative predictive values) as well as the assessment of the diagnostic performance for all these criteria combined (Table 3).

\section{Shear wave elastography (SWE) findings}

Each nodule underwent minimum, maximum, and mean SWE measurements three times, and the average of these measurement was calculated for each index. All of the three measurements were higher among the malignant nodules compared to their benign counterparts with a $p$ value of $<0.001$ (Table 4).

The optimal cut-off value of the minimum, maximum, and mean SWE measurements to differentiate between benign and malignant nodules with the highest sensitivity, specificity, and accuracy was determined using receiver operating curve (ROC) analysis. 
Table 2 Conventional ultrasound and Doppler criteria to detect malignant nodule ${ }^{(n=72)}$

\begin{tabular}{|c|c|c|c|c|c|}
\hline \multirow[t]{2}{*}{ Ultrasound features } & \multirow{2}{*}{$\begin{array}{l}\text { Malignant }(n=14) \\
N(\%)\end{array}$} & \multirow{2}{*}{$\begin{array}{l}\text { Benign }(n=58) \\
N(\%)\end{array}$} & \multicolumn{3}{|c|}{ Statistical significance } \\
\hline & & & $p$ value* & Odds ratio $(95 \% \mathrm{Cl})$ & $\begin{array}{l}\text { Phi } \\
\text { coefficiency } \\
\#\end{array}$ \\
\hline \multicolumn{6}{|l|}{ Echopattern } \\
\hline Hypoechoic & $10(71.4)$ & $9(15.5)$ & $<0.001$ & $13.61(3.49-53.03)$ & 0.502 \\
\hline Not hypoechoic & $4(28.6)$ & $49(84.5)$ & & & \\
\hline \multicolumn{6}{|l|}{ Shape } \\
\hline Taller than wide & $9(64.3)$ & $19(32.8)$ & 0.037 & $3.695(1.09-12.55)$ & 0.256 \\
\hline Wider than tall & $5(35.7)$ & $39(67.2)$ & & & \\
\hline \multicolumn{6}{|l|}{ Consistency } \\
\hline Solid & $12(85.7)$ & $47(81.0)$ & 1.0 & $1.4(0.27-7.2)$ & .048 \\
\hline Partially solid & $2(14.3)$ & $11(19.0)$ & & & \\
\hline \multicolumn{6}{|l|}{ Halo } \\
\hline Halo Absent & $12(85.7)$ & $11(19.0)$ & $<0.001$ & $25.64(5.0-131.44)$ & 0.567 \\
\hline Halo Present & $2(14.3)$ & $47(81.0)$ & & & \\
\hline \multicolumn{6}{|l|}{ Outline } \\
\hline Irregular & $8(57.1)$ & $8(13.8)$ & 0.002 & $8.333(2.28-30.43)$ & .413 \\
\hline Regular & $6(42.9)$ & $50(86.2)$ & & & \\
\hline \multicolumn{6}{|l|}{ Calcification } \\
\hline Micro-calcification & $11(78.6)$ & $2(3.4)$ & $<0.001$ & $102.67(15.32-688.1)$ & 0.773 \\
\hline No mico-calcification & $3(21.4)$ & $56(96.6)$ & & & \\
\hline \multicolumn{6}{|l|}{ Vascularity } \\
\hline Internal vascularity \&/internal and peripheral & $12(85.7)$ & $6(10.3)$ & $<0.001$ & $52.0(9.32-290.11)$ & 0.689 \\
\hline No vascularity and/peripheral vascularity & $2(14.3)$ & $52(89.7)$ & & & \\
\hline
\end{tabular}

*Fisher exact test was used as some expected cell values were less than 5

$\mathrm{Cl}$, Confidence interval

Significant $p$ value if $<0.05$ level

\# To detect effect size of the difference

Weak (0.2)-Moderate (0.4)-Strong (0.6)-Very strong (0.8)-Perfect (1.0)

Table 3 Diagnostic performance of each conventional ultrasound and Doppler finding to detect malignant nodules $(\mathrm{n}=72)$

\begin{tabular}{llllll}
\hline Ultrasound features & Sensitivity \% $\mathbf{9 5} \% \mathbf{C l})$ & Specificity \% $\mathbf{( 9 5 \% ~ C l )}$ & Accuracy \% (95\% Cl) & PPV \% (95\% Cl) & NPV \% (95\% Cl) \\
\hline Hypoechoic echopattern & $71.43(41.9-91.61)$ & $84.48(72.58-92.65)$ & $81.94(71.11-90.02)$ & $52.63(35.88-68.81)$ & $92.45(84.16-96.58)$ \\
Taller than wide & $64.29(35.14-87.24)$ & $67.24(53.66-78.99)$ & $66.67(54.57-77.34)$ & $32.14(21.68-44.76)$ & $88.64(79.06-94.16)$ \\
Halo Absent & $85.71(57.19-98.22)$ & $81.03(68.59-90.13)$ & $81.94(71.11-90.02)$ & $52.17(38.08-65.93)$ & $95.92(86.62-98.84)$ \\
Irregular & $57.14(28.86-82.34)$ & $86.21(74.62-93.85)$ & $80.56(69.53-88.64)$ & $50.0(31.28-68.72)$ & $89.29(81.86-93.9)$ \\
Micro-calcification & $78.57(49.2-96.34)$ & $96.55(88.09-99.58)$ & $93.06(84.53-97.71)$ & $84.62(57.83-95.66)$ & $94.92(87.24-98.08)$ \\
Internal Vascularity & $85.71(57.19-98.22)$ & $89.66(78.83-96.11)$ & $88.89(79.28-95.08)$ & $66.67(47.65-81.46)$ & $96.30(87.78-98.85)$ \\
Total* $^{*}$ & $94.2(73.2-99.8)$ & $69.89(59.3-81.85)$ & $74.62(61.7-85.9)$ & $83.21(54.5-95.9)$ & $78.9(63.7-87.6)$ \\
\hline
\end{tabular}

$P P V$, positive predictive value; NPV (negative predictive value

*Total include all above criteria

We identified the values of $42.2 \mathrm{kPa}$ for mean SWE (area under curve, 0.921 ), $53.6 \mathrm{kPa}$ for maximum SWE (area under curve, 0.895), and $26.9 \mathrm{kPa}$ for minimum SWE (area under curve, 0.906) (Table 5) as the best cutoff values to distinguish between benign and malignant nodules. The diagnostic performance of each measurement is clarified in Table 6 . Out of the three cut-off values, the mean SWE (42.2 $\mathrm{kPa})$ shows the best sensitivity $(85.71 \%)$, specificity $(94.83 \%)$, accuracy $(93.06 \%)$, and PPV (76.9\%), while the maximum SWE (53.6 kPa) shows the best NPV (94.7\%). 
Table 4 Mean values of SWE waves according to histopathology findings among the studied group

\begin{tabular}{llll}
\hline Parameter & Malignant & Benign & $\boldsymbol{p}$ value \\
\hline SWE- Mean & $49.71 \pm 11.16$ & $33.46 \pm 5.02$ & $<0.001$ \\
SWE- Minimum & $35.5 \pm 8.79$ & $22.19 \pm 4.39$ & $<0.001$ \\
SWE- Maximum & $63.91 \pm 13.87$ & $44.73 \pm 5.89$ & $<0.001$ \\
\hline
\end{tabular}

*Independent samples t-test was used

Table 5 Optimal cut-off values of SWE parameters

\begin{tabular}{lll}
\hline Parameter & AUC $(\mathbf{9 5 \%} \mathrm{Cl})$ & $\begin{array}{l}\text { Optimal } \\
\text { cut-off } \\
\text { values }\end{array}$ \\
\hline SWE-mean & $0.921(0.832-0.971)$ & 42.2 \\
SWE-minimum & $0.906(0.814-0.962)$ & 26.9 \\
SWE-maximum & $0.895(0.801-0.955)$ & 53.6 \\
\hline
\end{tabular}

Furthermore, we studied the diagnostic performance of the combined use of all ultrasound criteria mentioned in Table 3 and the mean SWE, and we found a significant increase in the diagnostic performance in comparison to using the ultrasound criteria only, but the diagnostic performance was variable when compared with mean SWE (sensitivity $96.3 \%$, specificity $89.8 \%$, accuracy $91.06 \%$, PPV 90.06\%, and NPV 88.3\%).

\section{Discussion}

Thyroid nodules, although uncommon among the pediatric population, pose a great challenge to pediatricians and pediatric surgeons. Among the pediatric population, the risk of a thyroid nodule being malignant may reach up to $18-26.7 \%$ [3].

Males and females are reported to have an equal proportion of thyroid malignancy in the pediatric age group. However, females may have a four-time prevalence risk compared to males at the post pubertal age [21]. The current study showed an apparently higher prevalence of thyroid nodule among females (64.3\%) compared to their male peers (35.7\%); this is concordant with previous studies $[4,22]$.

The current widely adopted methods of ultrasonographic differentiation between benign and malignant thyroid nodules rely mainly on certain sonographic criteria that have the highest summation of sensitivity and specificity as well as the highest accuracy [23, 24]. Many authors have reported that using a combination of multiple ultrasound criteria are necessary for diagnosis, and they consider that the combination of four ultrasonographic criteria improves the diagnostic accuracy to detect malignant thyroid nodules [23-25].

Moon et al. [23] assessed the diagnostic accuracy of US for differentiation between benign and malignant thyroid nodules and found that the US criteria including irregular shape, hypoechogenicity, taller-than-wide dimensions, and microcalcifications, were helpful for the discrimination of benign from malignant thyroid nodules. These criteria had high sensitivity for diagnosing malignant nodule $(80 \%, 81.3 \%, 75.3 \%$, and $74.7 \%$, respectively). However, this study had a low diagnostic accuracy, which could be explained by the study not using vascularity criteria in their assessment, which proved to be an important feature for the prediction of malignancy in our study.

In the current study, multiple sonographic criteria were assessed for their utility in differentiation between benign and malignant thyroid nodules according to the ATA (American Thyroid Association) guidelines. The criteria that were most predictive of malignancy were the presence of microcalcifications in $11 / 14$ (78.6\%) with $93.06 \%$ accuracy, internal vascularity or mixture of internal and peripheral vascularity in 12 out of 14 malignant nodules $(85.7 \%)$ with $88.89 \%$ accuracy, absence of a halo around the nodule in 12 out of 14 malignant nodules (85.7\%) with $81.94 \%$ accuracy, and hypoechogenicity in $10 / 14(71.4 \%)$ with $81.94 \%$ accuracy. All these criteria are in accordance with the American Thyroid Association (ATA) guidelines and display higher accuracy in the characterization of thyroid nodules in children and adolescents than in adults.

Other criteria were also relatively predictive, albeit with a lower accuracy, including an irregular outline in $8 / 14$ (57.1\%) with $80.56 \%$ accuracy and longitudinal appearance rather than wideness in $9 / 14$ (64.3\%) with $66.67 \%$ accuracy. Using a combination of these criteria for the detection of malignancy resulted in $94.2 \%$ sensitivity, $69.89 \%$ specificity, $74.62 \%$ accuracy, $83.21 \%$ positive predictive, and $78.9 \%$ negative predictive values.

Table 6 Diagnostic performance of shear wave elastography to detect malignant nodules $(n=72)$

\begin{tabular}{|c|c|c|c|c|c|}
\hline SWE & Sensitivity $(95 \% \mathrm{CI})$ & Specificity $(95 \% \mathrm{Cl})$ & Accuracy $(95 \% \mathrm{Cl})$ & PPV $(95 \% \mathrm{Cl})$ & NPV $(95 \% \mathrm{Cl})$ \\
\hline SWE-Mean & $85.71 \%$ (57.2-98.2) & $94.83 \%(85.6-98.9)$ & $93.06 \%(85.5-96.8)$ & $76.9 \%$ (51.3-91.3) & $93.2 \%(85.7-96.9)$ \\
\hline SWE-Minimum & $71.43 \%$ (41.9-91.6) & $86.21 \%(74.5-93.9)$ & $83.34 \%(79.9-86.4)$ & $55.6 \%(37.7-72.0)$ & $92.6 \%(84.4-96.6)$ \\
\hline SWE-Maximum & $78 . .57 \%(49.2-95.3)$ & $93.10 \%(83.3-98.1)$ & $90.28 \%(83.2-94.4)$ & $73.3 \%(50.7-88.0)$ & $94.7 \%(86.8-98.0)$ \\
\hline
\end{tabular}

PPV (positive predictive value). NPV (negative predictive value) 
These data are in accordance with another study that reported $87.0 \%$ sensitivity and $86.5 \%$ specificity when using high resolution ultrasonography [10].

Other studies also are concordant with our results $[10,14]$ and highlight the importance of high-resolution ultrasound (HRUS) in diagnosing thyroid nodules. Rago et al. [26] found that the presence of microcalcifications combined with the absence of a halo sign was highly predictive of malignancy. Takashima et al. [27] concluded that microcalcifications had the greatest accuracy $(76 \%)$ and highest specificity (93\%) for the diagnosis of malignant nodules. However, another report by Shuzhen et al. [28] contradicted these results by showing different results, including a higher sensitivity and a lower specificity of HRUS.

The current study adopted SWE to discriminate between benign and malignant thyroid nodules among children and adolescents as this has not been extensively investigated before. We reported a statistically significant difference among SWE measurements including mean, minimum, and maximum values $(p<0.001)$, highlighting the utility of SWE in this regard, as has been previously documented in adults [13-15].

The cut-off values used as the best index to differentiate benign from malignant thyroid nodules are still under debate, with wide variation between different reports, ranging between a mean valu/e of $85.2 \mathrm{kPa}(43.6 \%$ sensitivity, $88.7 \%$ specificity, 93.8\% PPV, and $28.7 \%$ NPV [29] and $34.5 \mathrm{kPa}(76.9 \%$ sensitivity and $71.7 \%$ specificity) [15]. Other studies reported SWE cut-off values between $65 \mathrm{kPa}$ with $85.2 \%$ sensitivity, $93.9 \%$ specificity, $80 \%$ positive predictive value, and $95.9 \%$ negative predictive value [13] and $66 \mathrm{kPa}$ with $80 \%$ sensitivity and $90.5 \%$ specificity [14].

The current work found the SWE cut-off value to be $42.2 \mathrm{kPa}$ with the highest accuracy reported yet (93.06\%). In accordance with these results, Liu et al. [30] reported an SWE cut-off value of $38.3 \mathrm{kPa}$ for discriminating between benign and malignant nodules.

The discriminative power of SWE proved to be superior to other conventional sonographic tools in detecting malignant thyroid nodule [31]. This notion was clarified in our study as the combination of grayscale and Doppler ultrasound criteria was $80.45 \%$ accurate. On the other hand, the accuracy of SWE was $93.06 \%$.

Despite the existence of many sonographic malignancy risk stratification systems, such as ACR-TIRADS, we do recommend considering an SWE of more than $42.2 \mathrm{kPa}$ as a suspicious criterion for malignancy; we also recommend performing FNA for any thyroid nodule classified as TR3 or above according to ACR TI-RADS with an SWE of more than $42.2 \mathrm{kPa}$.
The diagnostic performance of the combination of the total suspicious sonographic criteria with the mean SWE may be superior to the sole sonographic criteria. However, this combination was variable when compared with the mean SWE (sensitivity $96.3 \%$, specificity $89.8 \%$, accuracy $91.06 \%$, PPV $90.06 \%$, and NPV $88.3 \%$ ).

Overall, the wide discrepancy between the different reports as regards the SWE cut-off value may be attributed to methodological differences among the studies. ROI application could be the main reason for such differences. Some studies used a fixed size to place it over the stiff part of the nodule to measure the stiffness, while others used different sizes of ROI to examine the stiffness throughout the nodule. Another reason for such variation may be due to the subjective judgment of the operator in placing the ROI. We applied a unified method for the ROI over the stiffest part of the nodule to differentiate the SWE cut-off point both for benign and malignant nodules.

Some limitations existed in the current study, including the low cohort number, which could be explained by low incidence of thyroid nodules in the pediatric age group in our setting. Additionally, the number of malignant nodules was low (14 nodules), and all of them were papillary carcinomas. Hence, we were not able to evaluate the difference of SWE indices among different types of malignant nodules. In addition, not all patients underwent surgical excision, which is the definitive method for diagnosing malignancy, in comparison to FNAB. Despite these limitations, this study contributes by highlighting the efficacy of SWE in the evaluation of children and adolescents' thyroid nodules.

\section{Conclusion}

SWE is an effective non-invasive technique that can help in the discrimination of benign and malignant thyroid nodules among children and adolescents. A cut-off value of $42.2 \mathrm{kPa}$ may be optimal in predictions of the likelihood of malignancy of thyroid nodules in this age group. Further studies with larger cohorts and standardized sonographic evaluation criteria incorporating SWE may allow the better management of children and adolescent thyroid nodules.

\footnotetext{
Abbreviations

ATA: American Thyroid association; FNAB: Fine needle aspiration biopsy; HRUS: High frequency ultrasound; IRB: Institutional review board; kPa: Kilopascal; SE: Sonoelastography; SWE: Shear wave elastography.

\section{Acknowledgements}

The authors acknowledge the Deanship of Scientific Research at King Faisal University for the financial support under Nasher Track (Grant No. 216104).
} 


\section{Methodology}

Prospective

Diagnostic or prognostic study

Performed at a single center.

\section{Authors' contributions}

The guarantor of this publication-(M.H). Study concept and design were contributed by (M.H., I.K.A.J., A.G.H., O.Z., M.Y.I.D., N.M.A.H). Literature research were contributed by (M.H., Abdulwahab A.A., A.G.H., Abdulrahim A.A., M.Q.A). Patients enrolment was contributed by (M.H., Abdulrahim A.A., O.Z., M.Y.I.D., M.Q.A). Data analysis was contributed by (M.H., I.K.A.J., Abdulwahab A.A., N.M.A.H.). Manuscript preparation was contributed by all authors. All authors read and approved the final manuscript.

\section{Funding}

This work supported under Nasher Track (Grant No. 216104).

\section{Availability of the data and materials}

The datasets used and/or analyzed during the current study are available from the corresponding author on reasonable request.

\section{Declarations}

\section{Ethical approval and consent to participate}

Ethical approval from our institutional review board (IRB) was obtained. Written informed consent was obtained from the patients above the age of 18 years, parents of the patients, or their legal guardians if less than 18 years.

\section{Consent for publication}

Not applicable.

\section{Competing of interest}

The authors declare that they have no competing interests.

\section{Author details}

${ }^{1}$ Department of Surgery, College of Medicine, King Faisal University, P.O. Box: 400, Al-Ahsa 31982, Kingdom of Saudi Arabia. ${ }^{2}$ Department of Radiology, Sohag Faculty of Medicine, Sohag University, Sohag, Egypt. ${ }^{3}$ Department of Surgery, Division of Pediatric Surgery, Faculty of Medicine, Suez Canal University, Ismailia, Egypt. ${ }^{4}$ Radiology Department, Polyclinic Center, King Faisal University, P.O. Box: 400, Al-Ahsa 31982, Kingdom of Saudi Arabia. ${ }^{5}$ Albaha University Medical Center, Albaha, Kingdom of Saudi Arabia. ${ }^{6}$ Maxillofacial Surgery Unit, Surgery Department, Sohag Faculty of Medicine, Sohag University, Sohag, Egypt. ${ }^{7}$ Department of Biomedical Sciences, College of Medicine, King Faisal University, Al Ahsa, Kingdom of Saudi Arabia. ${ }^{8}$ Department of Radiology, King Fahd Hospital Hofuf, Al Ahsa, Kingdom of Saudi Arabia. ${ }^{9}$ Sohag University Hospital, Sohag, Egypt.

Received: 6 March 2021 Accepted: 11 Auqust 2021 Published online: 09 September 2021

\section{References}

1. Koltin D, O'Gorman CS, Murphy A et al (2016) Pediatric thyroid nodules: ultrasonographic characteristics and inter-observer variability in prediction of malignancy. J Pediatr Endocrinol Metab 29:789-794. https://doi. org/10.1515/jpem-2015-0242

2. Francis GL, Waguespack SG, Bauer AJ et al (2015) Management guidelines for children with thyroid nodules and differentiated thyroid cancer. Thyroid 25:716-759. https://doi.org/10.1089/thy.2014.0460

3. Gupta A, Ly S, Castroneves LA et al (2013) A standardized assessment of thyroid nodules in children confirms higher cancer prevalence than in adults. J Clin Endocrinol Metab 98:3238-3245. https://doi.org/10.1210/jc. 2013-1796

4. Chaukar DA, Rangarajan V, Nair N et al (2005) Pediatric thyroid cancer. J Surg Oncol 92:130-133. https://doi.org/10.1002/jso.20339
5. Haugen BR, Alexander EK, Bible KC et al (2016) 2015 american thyroid association management guidelines for adult patients with thyroid nodules and differentiated thyroid cancer: the american thyroid association guidelines task force on thyroid nodules and differentiated thyroid cancer. Thyroid 26:1-133. https://doi.org/10.1089/thy.2015. 0020

6. Al Nofal A, Gionfriddo MR, Javed A et al (2016) Accuracy of thyroid nodule sonography for the detection of thyroid cancer in children: systematic review and meta-analysis. Clin Endocrinol (Oxf) 84:423-430. https://doi.org/10.1111/cen.12786

7. Redman R, Zalaznick H, Mazzaferri EL, Massoll NA (2006) The impact of assessing specimen adequacy and number of needle passes for fineneedle aspiration biopsy of thyroid nodules. Thyroid 16:55-60. https:// doi.org/10.1089/thy.2006.16.55

8. Zakaria OM, Mousa A, AlSadhan R et al (2018) Reliability of sonoelastography in predicting pediatric cervical lymph node malignancy. Pediatr Surg Int 34:885-890. https://doi.org/10.1007/s00383-018-4301-x

9. de Fleury E, FC, Fleury JCV, Oliveira VM de, et al (2009) Proposal for the systematization of the elastographic study of mammary lesions through ultrasound scan. Rev Assoc Med Bras 55:192-196. https://doi. org/10.1590/s0104-42302009000200025

10. Ragazzoni F, Deandrea M, Mormile A et al (2012) High diagnostic accuracy and interobserver reliability of real-time elastography in the evaluation of thyroid nodules. Ultrasound Med Biol 38:1154-1162. https://doi.org/10.1016/j.ultrasmedbio.2012.02.025

11. Athanasiou A, Tardivon A, Tanter M et al (2010) Breast lesions: quantitative elastography with supersonic shear imaging-preliminary results. Radiology 256:297-303. https://doi.org/10.1148/radiol.10090385

12. Sun J, Cai J, Wang X (2014) Real-time ultrasound elastography for differentiation of benign and malignant thyroid nodules: a meta-analysis. J Ultrasound Med 33:495-502. https://doi.org/10.7863/ultra.33.3.495

13. Sebag F, Vaillant-Lombard J, Berbis J et al (2010) Shear wave elastography: a new ultrasound imaging mode for the differential diagnosis of benign and malignant thyroid nodules. J Clin Endocrinol Metab 95:5281-5288. https://doi.org/10.1210/jc.2010-0766

14. Veyrieres J-B, Albarel F, Lombard JV et al (2012) A threshold value in Shear Wave elastography to rule out malignant thyroid nodules: a reality? Eur J Radiol 81:3965-3972. https://doi.org/10.1016/j.ejrad.2012.09. 002

15. Bhatia KSS, Tong CSL, Cho CCM et al (2012) Shear wave elastography of thyroid nodules in routine clinical practice: preliminary observations and utility for detecting malignancy. Eur Radiol 22:2397-2406. https://doi.org/ 10.1007/s00330-012-2495-1

16. Uysal E, Öztürk M (2019) Quantitative assessment of thyroid glands in healthy children with shear wave elastography. Ultrasound Q 35:297-300. https://doi.org/10.1097/RUQ.0000000000000426

17. Cunha GB, Marino LCl, Yamaya A et al (2019) Elastography for the evaluation of thyroid nodules in pediatric patients. Radiol Bras 52:141-147. https://doi.org/10.1590/0100-3984.2018.0034

18. Borysewicz-Sanczyk H, Dzieciol J, Sawicka B, Bossowski A (2016) Practical application of elastography in the diagnosis of thyroid nodules in children and adolescents. Horm Res Paediatr 86:39-44. https://doi.org/10. $1159 / 000447490$

19. Nascimento C, Borget I, Al Ghuzlan A et al (2015) Postoperative fluorine18-fluorodeoxyglucose positron emission tomography/computed tomography: an important imaging modality in patients with aggressive histology of differentiated thyroid cancer. Thyroid 25:437-444. https://doi. org/10.1089/thy.2014.0320

20. Cibas ES, Ali SZ (2017) The 2017 bethesda system for reporting thyroid cytopathology. Thyroid 27:1341-1346. https://doi.org/10.1089/thy.2017 0500

21. Hogan AR, Zhuge Y, Perez EA et al (2009) Pediatric thyroid carcinoma: incidence and outcomes in 1753 patients. J Surg Res 156:167-172. https://doi.org/10.1016/j.jss.2009.03.098

22. Essenmacher AC, Joyce PHJ, Kao SC, et al (2017) Sonographic Evaluation of Pediatric Thyroid Nodules. Radiographics 37:1731-1752. https://doi. org/10.1148/rg.2017170059

23. Moon W-J, Jung SL, Lee JH et al (2008) Benign and malignant thyroid nodules: US differentiation-multicenter retrospective study. Radiology 247:762-770. https://doi.org/10.1148/radiol.2473070944 
24. Paschke R, Hegedüs $L$, Alexander E et al (2011) Thyroid nodule guidelines: agreement, disagreement and need for future research. Nat Rev Endocrinol 7:354-361. https://doi.org/10.1038/nrendo.2011.1

25. Bo YH, Ahn HY, Lee YH et al (2011) Malignancy rate in sonographically suspicious thyroid nodules of less than a centimeter in size does not decrease with decreasing size. J Korean Med Sci 26:237-242. https://doi. org/10.3346/jkms.2011.26.2.237

26. Rago T, Santini F, Scutari M et al (2007) Elastography: new developments in ultrasound for predicting malignancy in thyroid nodules. J Clin Endocrinol Metab 92:2917-2922. https://doi.org/10.1210/jc.2007-0641

27. Takashima S, Fukuda H, Nomura N et al (1995) Thyroid nodules: Reevaluation with ultrasound. J Clin Ultrasound 23:179-184. https://doi.org/ $10.1002 / j$ jcu.1870230306

28. Shuzhen C (2012) Comparison analysis between conventional ultrasonography and ultrasound elastography of thyroid nodules. Eur J Radiol 81:1806-1811. https://doi.org/10.1016/j.ejrad.2011.02.070
29. Park AY, Son EJ, Han K et al (2015) Shear wave elastography of thyroid nodules for the prediction of malignancy in a large scale study. Eur J Radiol 84:407-412. https://doi.org/10.1016/j.ejrad.2014.11.019

30. Liu B-X, Xie X-Y, Liang J-Y et al (2014) Shear wave elastography versus realtime elastography on evaluation thyroid nodules: a preliminary study. Eur J Radiol 83:1135-1143. https://doi.org/10.1016/j.ejrad.2014.02.024

31. Duan S-B, Yu J, Li X et al (2016) Diagnostic value of two-dimensional shear wave elastography in papillary thyroid microcarcinoma. Onco Targets Ther 9:1311-1317. https://doi.org/10.2147/OTT.S98583

\section{Publisher's Note}

Springer Nature remains neutral with regard to jurisdictional claims in published maps and institutional affiliations.

\section{Submit your manuscript to a SpringerOpen ${ }^{\odot}$ journal and benefit from:}

- Convenient online submission

- Rigorous peer review

- Open access: articles freely available online

- High visibility within the field

Retaining the copyright to your article

Submit your next manuscript at $\boldsymbol{\nabla}$ springeropen.com 